\title{
РЕДУКТИВНЫЕ СТРУКТУРЫ И ТРОЙНЫЕ СИСТЕМЫ ЛИ
}

\author{
(Представил А. Хумал)
}

\section{1. Вводные понятия}

Пусть $G$ - связная группа Ли с алгеброй Ли g и $H-$ замкнутая подгруппа в $G$ с подалгеброй Ли $\mathfrak{h}$. Если в g существует такое подпространство $\mathrm{m}$, что $\mathfrak{g}=\mathfrak{h}+\mathfrak{m}$ (прямая сумма подпространств) и $A d H(m) \subset \mathrm{m}$ (локально $[\mathfrak{h}, \mathfrak{m}] \subset \mathrm{m})$, то говорят, что на однородном пространстве $G / H$ задана редуктивная спруктура $(\mathfrak{g}, \mathfrak{h}, \mathfrak{m})\left[{ }^{1,2}\right]$. В дальнейшем, говоря о редуктивной структуре $(\mathfrak{g}, \mathfrak{h}, \mathfrak{m})$, мы будем иметь в виду редуктивную структуру на соответствующем однородном пространстве $G / H$. Редуктивная структура $(\mathfrak{g}, \mathfrak{h}, \mathfrak{m})$ называется (локально) симметрической $\left[{ }^{2}\right]$, если $[\mathrm{m}, \mathrm{m}] \subset \mathfrak{h}$.

Следуя $\left[{ }^{3}\right]$, будем называть общей тройной системой (о. т. с.) над полем $k$ конечномерное векторное пространство $L$ над полем $k$, на котором заданы две алгебраические операции - билинейная и трилинейная

$$
\begin{array}{ll}
L \times L \rightarrow L, & (X, Y) \rightarrow X \cdot Y, \\
L X L \times L \rightarrow L, & (X, Y, Z) \rightarrow[X, Y, Z],
\end{array}
$$

причем выполняются следующие условия

$$
\begin{aligned}
& X \cdot X=0, \\
& {[X, X, Y]=0,} \\
& {[X, Y, Z]+[Y, Z, X]+[Z, X, Y]-(X \cdot Y) \cdot Z-(Y \cdot Z) \cdot X-(Z \cdot X) \cdot Y=0,} \\
& {[X, Y,[Z, V, W]]=[[X, Y, Z], V, W]+} \\
& +[Z,[X, Y, V], W]+[Z, V,[X, Y, W]], \\
& {[X \cdot Y, Z, V]+[Y \cdot Z, X, V]+[Z \cdot X, Y, V]=0,} \\
& {[X, Y, Z \cdot V]+V \cdot[X, Y, Z]+Z \cdot[X, Y, V]=0}
\end{aligned}
$$

для $X, Y, Z, V, W \in L$.

Конечномерная алгебра Ли g становится о. т. с., если для $X, Y, Z \in$ $\in \mathfrak{g}$ положить

$$
X \cdot Y=[X, Y], \quad[X, Y, Z]=[[X, Y], Z] .
$$

С каждой редуктивной структурой на однородном пространстве связана некоторая о. т. с. в силу следующего результата. 
Т е о рем а 1. $\left(\left[{ }^{4}\right]\right)$. Пусть $(\mathfrak{g}, \mathfrak{h}, \mathrm{m})$ - редуктивная структура на однородном пространстве G/H. Если на векторном пространстве м определить билинейную и трилинейную операции формулами

$$
X \cdot Y=[X, Y]_{\mathfrak{m}}, \quad[X, Y, Z]=(1 / 4)\left[[X, Y]_{\mathfrak{f}}, Z\right],
$$

где индексы m $u$ h обозначают проекцию в соответствующем подпространстве *, то п становится о. т. с.

О. т. с. m с введенными в теореме 1 операциями называется о. т. с. редуктивной структуры $(\mathfrak{g}, \mathfrak{h}, \mathrm{m})$.

Одновременно бнлинейная композиция в о. т. с. m определяет некоторую неассоциативную алгебру $(\mathrm{m}, \cdot)$, впервые рассмотренную А. Сэйглом в $\left[{ }^{5}\right]$.

В настоящей работе вводится понятие подструктуры редуктивной структуры и доказывается, что нахождение таких подструктур равносильно нахождению подсистем в о. т. с. этой структуры. Исследуется также взаимосвязь между вполне геодезическими подмногообразиями естественно-редуктивного однородного пространства и тройными системами Ли в соответствующей алгебре. Приводится аналог известного результата Э. Картана о связи между вполне геодезическими подмногообразиями симметрического пространства и тройными системами Ли.

\section{2. Подструктуры редуктивной структуры}

Пусть $(\mathfrak{g}, \mathfrak{h}, \mathfrak{m})$ - редуктивная структура. Следуя $\left[{ }^{6}\right]$, назовем подалгебру $\mathrm{g}^{\prime} \subset \mathrm{g}$ нормальной в $\mathfrak{g}$, если

$$
\mathrm{g}^{\prime}=\mathrm{g}^{\prime} \cap \mathfrak{h}+\mathrm{g}^{\prime} \cap \mathrm{m}
$$

О пределение 1. Тройку $\left(\mathfrak{g}^{\prime}, \mathfrak{h}^{\prime}, \mathrm{m}^{\prime}\right)$ назовем подструктурой редуктивной структуры $(\mathfrak{g}, \mathfrak{h}, \mathrm{m})$, если $\mathfrak{g}^{\prime}-$ нормальная подалгебра в $\mathfrak{g}$, причем $\mathfrak{h}^{\prime}=\mathfrak{g}^{\prime} \cap \mathfrak{h} u \mathfrak{m}^{\prime}=\mathfrak{g}^{\prime} \cap \mathrm{m}$. Если, кроме того, $\mathfrak{g}^{\prime}-$ идеал в $\mathfrak{g}$, то подструктуру $\left(\mathfrak{g}^{\prime}, \mathfrak{h}^{\prime}, \mathrm{m}^{\prime}\right)$ будем называть идеальной.

Так как $\left[\mathfrak{h}^{\prime}, \mathfrak{m}^{\prime}\right] \subset \mathfrak{g}^{\prime} \cap \mathfrak{m}=\mathfrak{m}^{\prime}$, то подструктура $\left(\mathfrak{g}^{\prime}, \mathfrak{h}^{\prime}, \mathfrak{m}^{\prime}\right)$ сама является редуктивной структурой и поэтому обладает о. т. с. $\mathrm{m}^{\prime}$.

О пр едел ение 2. Векторное подпространство п в о. т. с. м называется подсистемой, если

$$
\mathfrak{n} \cdot \mathfrak{n} \subset \mathrm{n}, \quad[\mathrm{n}, \mathrm{n}, \mathrm{n}] \subset \mathrm{n},
$$

и идеалом, если

$$
\mathrm{n} \cdot \mathrm{m} \subset \mathrm{n}, \quad[\mathrm{n}, \mathrm{m}, \mathrm{m}] \subset \mathrm{n} .
$$

Те орем а 2. Пусть (g, h, m) - редуктивная структура и а) Если $\left(\mathfrak{g}^{\prime} \mathfrak{h}^{\prime}, \mathfrak{m}^{\prime}\right)$ - подструктура структуры (g, h, m), то ее о. т. с. $\mathfrak{m}^{\prime}$ является подсистемой в $\mathrm{m}$.

б) Для всякой подсистемы $\mathrm{m}^{\prime}$ в о. т. с. м существует такая подструктура $\left(\mathfrak{g}^{\prime}, \mathfrak{h}^{\prime}, \mathrm{m}^{\prime}\right)$ структуры $(\mathfrak{g}, \mathfrak{h}, \mathrm{m})$, для которой $\mathrm{m}^{\prime}$ является ее о. т. $с$. Д ок а з а тельст в о. а) Покажем, что $\mathfrak{m}^{\prime} \cdot \mathfrak{m}^{\prime} \subset \mathfrak{m}^{\prime}$ и $\left[\left[\mathfrak{m}^{\prime}, \mathfrak{m}^{\prime}\right]_{\mathfrak{l}}, \mathfrak{m}^{\prime}\right] \subset$

* В дальнейшем проекцию вектора или подпространства из $\mathfrak{g}$ на $\mathfrak{\text { g }}$ (соответственно на m) будем обозначать индексом h (соответственно m). 
$\subset \mathrm{m}^{\prime}$. Произведение $\mathrm{m}^{\prime} \cdot \mathrm{m}^{\prime}$ содержится в $\mathrm{m}$ (по определению) и в $\mathrm{g}^{\prime}$ (так как $\mathrm{g}^{\prime}-$ подалгебра в $\mathrm{g}$ ), поэтому $\mathrm{m}^{\prime} \cdot \mathrm{m}^{\prime} \subset \mathrm{m}^{\prime} \mathrm{g}^{\prime}=\mathrm{m}^{\prime}$, тем самым первое утверждение доказано. Далее, $\left[\mathfrak{m}^{\prime}, \mathfrak{m}^{\prime}\right]_{\mathfrak{h}} \subset \mathfrak{h}$ и $\left[\mathfrak{m}^{\prime}, \mathfrak{m}^{\prime}\right]_{\mathfrak{h}} \subset$ $\subset \mathfrak{g}^{\prime}$, следовательно, $\left[\mathfrak{m}^{\prime}, \mathfrak{m}^{\prime}\right]_{\mathfrak{h}} \subset \mathfrak{h} \cap \mathfrak{g}^{\prime}=\mathfrak{h}^{\prime}$. Но тогда $\left[\left[\mathfrak{m}^{\prime}, \mathfrak{m}^{\prime}\right]_{\mathfrak{h}}, \mathfrak{m}^{\prime}\right] \subset$ $\subset \mathfrak{m}^{\prime}$, так как $\left(\mathfrak{g}^{\prime}, \mathfrak{h}^{\prime}, \mathfrak{m}^{\prime}\right)$ - редуктивная структура, что доказывает и второе утверждение.

б) Положим $\mathrm{g}^{\prime}=\left[\mathfrak{m}^{\prime}, \mathfrak{m}^{\prime}\right]_{\mathfrak{h}}+\mathfrak{m}^{\prime}$. Для $X, Y, Z, V \in \mathfrak{m}^{\prime}$ имеем

$$
\begin{gathered}
{[X, Y]=[X, Y]_{\mathfrak{h}}+X \cdot Y \in \mathfrak{g}^{\prime},} \\
{\left[[X, Y]_{\mathfrak{h}}, Z\right] \in \mathrm{m}^{\prime} \subset \mathfrak{g}^{\prime},}
\end{gathered}
$$

так как $\mathrm{m}^{\prime}$ - подсистема в $m$. Далее,

$$
\begin{gathered}
{\left[[X, Y]_{\mathfrak{h}},[Z, V]_{\mathfrak{h}}\right]=\left[[X, Y]_{\mathfrak{h}},[Z, V]\right]-\left[[X, Y]_{\mathfrak{h}}, Z \cdot V\right] ;} \\
{\left[[X, Y]_{\mathfrak{h}}, Z \cdot V\right] \in \mathfrak{m}^{\prime} \subset \mathfrak{g}^{\prime} ;} \\
{\left[[X, Y]_{\mathfrak{h}},[Z, V]\right]=-\left[Z,\left[V,[X, Y]_{\mathfrak{h}}\right]\right]-\left[V,\left[[X, Y]_{\mathfrak{h}}, Z\right]\right]=} \\
=-\left[Z,\left[V,[X, Y]_{\mathfrak{h}}\right]\right]_{\mathfrak{h}}-\left[Z,\left[V,[X, Y]_{\mathfrak{h}}\right]\right]_{\mathfrak{m}}- \\
-\left[V,\left[[X, Y]_{\mathfrak{h}}, Z\right]\right]_{\mathfrak{h}}-\left[V,\left[[X, Y]_{\mathfrak{h}}, Z\right]\right]_{\mathfrak{m}} \in\left[\mathrm{m}^{\prime}, \mathrm{m}^{\prime}\right]_{\mathfrak{h}}+\mathfrak{m}^{\prime},
\end{gathered}
$$

так как $\mathfrak{m}^{\prime}$ - подсистема в $\mathfrak{g}^{\prime}$ - подалгебра в $\mathfrak{g}$ и подструктура $\left(\mathfrak{g}^{\prime},\left[\mathrm{m}^{\prime}, \mathrm{m}^{\prime}\right]_{\mathfrak{h}}, \mathrm{m}^{\prime}\right)$ - искомая.

Т е орем а 3. Пусть (g, h, m) - редуктивная структура, $\mathfrak{m}-$ eе o. T. c. $u \mathfrak{h}=[\mathrm{m}, \mathfrak{m}]_{\mathfrak{h}}$.

а) Если $\left(\mathfrak{g}^{\prime}, \mathfrak{h}^{\prime}, \mathfrak{m}^{\prime}\right)$ - идеальная подструктура структуры (g, h, $\left.\mathfrak{m}\right)$, то ее о. т. с. ' $\mathbf{\prime}^{\prime}$ есть идеал в $\mathrm{m}$.

б) Обратно: для всякого идеала $\mathrm{m}^{\prime}$ в о. т. с. м существует такая идеальная подструктура $\left(\mathfrak{g}^{\prime}, \mathfrak{h}^{\prime}, \mathrm{m}^{\prime}\right)$ структуры $(\mathfrak{g}, \mathfrak{h}, \mathrm{m})$, для которой $\mathfrak{m}^{\prime}$ является ее о. т. с.

Доказ а тельство. а) Покажем, что $\mathfrak{m}^{\prime} \cdot \mathfrak{m} \subset \mathfrak{m}^{\prime}$ и $\left[\left[\mathrm{m}^{\prime}, \mathfrak{m}\right]_{\mathfrak{h}}, \mathfrak{m}\right] \subset$ $\subset \mathfrak{m}^{\prime}$. Действительно, для $X \in \mathfrak{m}^{\prime}, \quad Y, Z \in \mathrm{m}$ имеем $[X, Y]=$ $=[X, Y]_{\mathfrak{h}}+X \cdot Y$. Произведение $X \cdot Y$ содержится в $m$ (по определению) и в $\mathrm{g}^{\prime}$ (так как $\mathrm{g}^{\prime}-$ идеал в $\mathrm{g}$ ), поэтому оно содержится и в их пересечении $\mathfrak{m}^{\prime}$. Далее, $[X, Y]_{\mathfrak{h}} \in \mathfrak{h} \cap \mathfrak{g}^{\prime}=\mathfrak{h}^{\prime} \quad$ и тогда $\left[[X, Y]_{\mathfrak{h}}, Z\right] \in \mathfrak{m}^{\prime}$ в силу редуктивности структуры $\left(\mathfrak{g}^{\prime}, \mathfrak{h}^{\prime}, \mathrm{m}^{\prime}\right)$.

б) Покажем, что подпространство $\mathrm{g}^{\prime}=\left[\mathrm{m}^{\prime}, \mathrm{m}\right]_{\mathfrak{h}}+\mathfrak{m}^{\prime}$ является идеалом в алгебре Ли g. Пусть $X, Y, Z \in \mathfrak{m}^{\prime}, V, W \in \mathfrak{m}, U \in \mathfrak{h}$. Тогда

$$
[X, V]=[X, V]_{\mathfrak{h}}+X \cdot V \in\left[\mathfrak{m}^{\prime}, m\right]_{\mathfrak{h}} f \mathfrak{m}^{\prime}=\mathfrak{g}^{\prime},
$$




$$
\left[[X, V]_{\mathfrak{h}}, W\right] \in \mathfrak{m}^{\prime} \subset \mathfrak{g}^{\prime},
$$

так как $\mathfrak{m}^{\prime}-$ идеал в о. т. с. $\mathfrak{m}$. Теперь, используя условие $\mathfrak{h}=$ $=[\mathrm{m}, \mathrm{m}]_{\mathfrak{h}}$, запишем

$$
\begin{gathered}
{\left[\mathfrak{h}, \mathfrak{m}^{\prime}\right]=\left[\left[\mathfrak{m}, \mathfrak{m}_{\mathfrak{h}}, \mathfrak{m}^{\prime}\right]=\left[[\mathfrak{m}, \mathfrak{m}]_{\mathfrak{h}}, \mathfrak{m}^{\prime}\right]_{\mathfrak{m}} \subseteq\right.} \\
\subseteq\left[[\mathfrak{m}, \mathfrak{m}], \mathfrak{m}^{\prime}\right]_{\mathfrak{m}} \subseteq\left[\left[\mathfrak{m}, \mathfrak{m}^{\prime}\right], \mathfrak{m}\right]_{\mathfrak{m}}= \\
=\left[\left[\mathfrak{m}, \mathfrak{m}^{\prime}\right]_{\mathfrak{h}}, \mathfrak{m}\right]_{\mathfrak{m}}+\left[\mathfrak{m}, \mathfrak{m}^{\prime}\right]_{\mathfrak{m}} \cdot \mathfrak{m} \subset \mathfrak{m}^{\prime},
\end{gathered}
$$

поэтому

$$
[U, X]=\left[[V, W]_{\mathfrak{h}}, X\right] \in \mathfrak{m}^{\prime} \subset \mathfrak{g}^{\prime} .
$$

В заключение докажем включение $\left[\mathfrak{h},\left[\mathfrak{m}^{\prime}, \mathfrak{m}\right]_{\mathfrak{h}}\right] \subset \mathfrak{g}^{\prime}$. Действительно, $[U,[X, V]]=\left[U,[X, V]_{\mathfrak{h}}\right]+[U, X \cdot V] . \quad$ Қак показано выше, скобка $[U, X \cdot V]$ содержится в $\mathfrak{m}^{\prime}$. Далее,

$$
\begin{gathered}
{[U,[X, V]]=-[X,[V, U]]-[V,[U, X]]=} \\
=-[X,[V, U]]_{\mathfrak{h}}-X \cdot[V, U]-[V,[U, X]]_{\mathfrak{h}}-V \cdot[U, X] \in \mathfrak{g}^{\prime},
\end{gathered}
$$

следовательно,

$$
\left[U,[X, V]_{\mathfrak{h}}\right] \in g^{\prime} .
$$

Из соотношений (6)-(9) следует, что $\mathfrak{g}^{\prime}-$ идеал в $g$ и подструктура $\left(\mathfrak{g}^{\prime},\left[\mathrm{m}^{\prime}, \mathrm{m}\right]_{\mathfrak{h}}, \mathrm{m}^{\prime}\right)$ - искомая.

\section{3. Естественно-редуктивные структуры и тройные системы Ли}

Пусть на алгебре Ли g задана билинейная симметрическая невырожденная $\operatorname{ad}(\mathrm{g})$-инвариантная форма $B$. Рассмотрим однородное пространство $G / H$, на котором редуктивная структура задается подпространством

$$
\mathfrak{m}=\{X \in \mathfrak{g} \mid B(X, Y)=0 \quad \forall Y \in \mathfrak{h}\},
$$

и предположим, что ограничение формы $B$ на подалгебре $\mathfrak{h}$ невырождено. Поскольку в этом случае для любых $X, Y, Z \in \mathrm{m}$ имеем

$$
\begin{aligned}
B(X \cdot Y, Z)= & B\left([X, Y]-[X, Y]_{\mathfrak{h}}, Z\right)=B([X, Y], Z)= \\
& =B(X,[Y, Z])=B(X, Y \cdot Z),
\end{aligned}
$$

то $G / H$ является естественно-редуктивным пространством $\left[{ }^{7}\right]$. Соответствуюшую структуру $(\mathfrak{g}, \mathfrak{h}, \mathfrak{m})$ будем называть естественно-редуктивной.

О п р ед ел е н и е 3. Подпространство п конечномерной вещественной алгебры Ли g называется тройной системой Ли (т. с. Ли) в g, если $[\mathrm{n},[\mathrm{n}, \mathrm{n}]] \subset \mathrm{n}$, 
Т е о р е а 4. Естественно-редуктивная структура (g, h, m) является симметрической тогда и только тогда, когда m является т. с. Ли.

Д ок а з а тельст во. Если (g, h, m) - симметрическая структура, то $[\mathrm{m}, \mathrm{m}] \subset \mathfrak{h}$ и поэтому $[\mathrm{m},[\mathrm{m}, \mathrm{m}]] \subset \mathrm{m}$. Обратно, пусть $\mathrm{m}-$ т. с. Ли. Тогда из равенств

$$
B([\mathfrak{h}, \mathrm{m}],[\mathrm{m}, \mathrm{m}])=B(\mathfrak{h},[\mathrm{m},[\mathrm{m}, \mathrm{m}]])=0
$$

следует $[\mathrm{m}, \mathrm{m}] \subset \mathfrak{h}$ и $(\mathfrak{g}, \mathfrak{h}, \mathfrak{m})-$ симметрическая структура.

О пр еделе ни е 4. Редуктивная структура (g, h), m) называется изотропно-неприводимой $\left[{ }^{2}\right]$, если i не содержит собственных аd h-инвариантных подпространств.

Л ем ма. Подструктура $\left(\mathfrak{g}^{\prime}, \mathfrak{h}^{\prime}, \mathrm{m}^{\prime}\right)$ естественно-редуктивной изотропно-неприводимой структуры (g, h), 1 ) является симметрической тогда и только тогда, когда $\mathrm{m}^{\prime}$ является т. с. Ли.

Д ок а з а т е л ь с т в . Если $\left(\mathfrak{g}^{\prime}, \mathfrak{h}^{\prime}, \mathrm{m}^{\prime}\right)$ - симметрическая структура, то $\left[\mathrm{m}^{\prime}, \mathrm{m}^{\prime}\right] \subset \mathfrak{h}^{\prime}$ и потому $\left[\mathrm{m}^{\prime},\left[\mathrm{m}^{\prime}, \mathrm{m}^{\prime}\right]\right] \subset \mathrm{m}^{\prime}$. Обратно, пусть $\mathrm{m}^{\prime}-$ т. с. Ли. В силу изотропной неприводимости структуры $(\mathfrak{g}, \mathfrak{h}, \mathfrak{m})$ имеем $\left[\mathfrak{h}, \mathfrak{m}^{\prime}\right]=\mathfrak{m}$ и тогда

$$
\begin{gathered}
B\left(\mathfrak{m},\left[\mathfrak{m}^{\prime}, \mathfrak{m}^{\prime}\right]\right)=B\left(\left[\mathfrak{h}, \mathfrak{m}^{\prime}\right],\left[\mathfrak{m}^{\prime}, \mathfrak{m}^{\prime}\right]\right)= \\
=B\left(\mathfrak{h},\left[\mathfrak{m}^{\prime},\left[\mathfrak{m}^{\prime}, \mathfrak{m}^{\prime}\right]\right]\right)=0,
\end{gathered}
$$

следовательно, $\left[\mathrm{m}^{\prime}, \mathrm{m}^{\prime}\right] \subset \mathfrak{h}$. Но $\mathfrak{g}^{\prime}-$ подалгебра в $\mathfrak{g}$, потому $\left[\mathrm{m}^{\prime}, \mathrm{m}^{\prime}\right] \subset \mathfrak{h} \cap \mathfrak{g}^{\prime}=\mathfrak{h}^{\prime}$.

Приводимая ниже теорема А. М. Васильева $\left[{ }^{6}\right]$, уточненная Я. Сенте $\left[{ }^{8}\right]$ на случай однородных пространств с редуктивной структурой, дает геометрическую характеристику подструктур редуктивной структуры.

Т еорем а 5. Пусть $(\mathfrak{g}, \mathfrak{h}, \mathrm{m})$ - редуктивная структура на $M=G / H$. Если касательное пространство $T_{o}(M) \kappa M$ в точке $о=H$ отождествить, как обычно с m, то однородное подмногообразие $M$, соответствующее подструктуре $\left(\mathfrak{g}^{\prime}, \mathfrak{h}^{\prime}, \mathrm{m}^{\prime}\right)$, является вполне геодезическим относительно естественной связности, индуцированной редуктивной структурой. Обратно: если $M^{\prime}$ - вполне геодезическое подмногообразие в $M, o \in M^{\prime}$ и все геодезические в $M^{\prime}$ являются траекториями однопараметрических подгрупп, порожденных элементами из $\mathrm{m}$, то существует редуктивная подструктура $\left(\mathfrak{g}^{\prime}, \mathfrak{h}^{\prime}, \mathrm{m}^{\prime}\right)$, для которой $T_{o}\left(M^{\prime}\right)=\mathfrak{m}^{\prime}$.

Следующая теорема, частично сформулированная в $\left[{ }^{9}\right]$, распространяет известный результат Э. Картана о связи между вполне геодезическими подмногообразиями симметрического пространства и т. с. Ли на однородные пространства с естественно-редуктивной структурой. Т ео р е а 6. Пусть (g, h, m) - естественно-редуктивная изотропнонеприводимая структура на $M=G / H u \mathrm{~m}$ - касательное пространство $T_{о}(M) \kappa M$ в точке $о=H$. Пусть, далее, s- т. с. Ли, содержащаяся в м. Тогда существует вполне геодезическое относительно естественной связности подмногообразие $M^{\prime}$ такое, что $T_{o}\left(M^{\prime}\right)=\xi$, причем в $M^{\prime}$ индуцируется симметрическая структура. Обратно: если $M^{\prime}$ - вполне геодезическое относительно естественной связности подмногообразие в М с симметрической структурой $и о \in M^{\prime}$, то существует т. с. Ли $\Xi \subset \sharp$ такая, ито $T_{o}\left(M^{\prime}\right)=\Xi$.

Д ок аз а тельство. Пусть $\xi-$ т. с. Ли. Тогда подпространство 


$$
\mathrm{g}^{\prime}=[\mathfrak{\xi}, \mathfrak{\xi}]+\mathfrak{z}
$$

является нормальной подалгеброй в g, задающей одновременно симметрическую структуру. По теореме 5 соответствующее $\mathrm{g}^{\prime}$ многообразие $M^{\prime}$ будет вполне геодезическим относительно естественной связности с симметрической структурой $\left(\mathfrak{g}^{\prime},[\xi, \xi], \xi\right)$.

Пусть, теперь, $M^{\prime}$ - вполне геодезическое относительно естественной связности подмногообразие с симметрической структурой и $o \in M^{\prime}$. Тогда все геодезические из $M^{\prime}$, проходящие через $о$, являются орбитами однопараметрических подгрупп, порожденных элементами из $\mathrm{m}$. Тем самым выполнены условия теоремы 5 и существует подструктура $\left(\mathfrak{g}^{\prime}, \mathfrak{h}^{\prime}, \mathfrak{g}\right)$ такая, что $T_{o}\left(M^{\prime}\right)=\mathfrak{s}$. В силу симметричности $M^{\prime}$ эта подструктура также будет симметрической, а подпространство $\$$, согласно лемме, - т. с. Ли.

\section{ЛИТЕРАТУРА}

1. Р а шевский П. К., В кн.: Труды семинара по векторному и тензорному анализу, вып. 9, М., Изд-во МГУ, 1952, с. 49-74.

2. N o m iz u, K., Amer. J. Math., 76, 33-65 (1954)

3. Y a m a g ut i, K., J. Sci. Hiroshima Univ., A21, № 2, 155-160 (1958).

4. S a g le, A., Nagoya Math. J., 32, June, 373-394 (1969).

5. S a g 1 e, A., J. Math. and Mech., 16, № 12, 1381-1393 (1967).

6. В а с и л в е в А. М., Докл. АН СССР, 128, № 2, 223-226 (1959).

7. Kobayashi, S., Nomizu, K., Foundation of Differential Geometry, 2, Interscience, New York-London, 1969, p. 210-316.

8. Sz e 11 the, J., Period. polytechn. Mech. Eng., 21, № 2, 111-128 (1977).

9. Ф ля й е р Ӓ., В кн.: Труды геометрического семинара, 6, М., изд. ВИНити, 1974 , c. $267-276$.

Информационно-вычислительный центр Министерства финансов ЭССР

Поступила в редакцию $22 /$ IV 1980

\section{A. FLJAISER}

\section{REDUKTIIVSED STRUKTUURID JA LIE KOLMIKSUSTEEMID}

Olgu $(\mathfrak{g}, \mathfrak{h}, \mathfrak{m})$ reduktiivne struktuur homogeensel ruumil $\mathrm{G} / \mathrm{H}\left[{ }^{1,2}\right]$ ja $\mathfrak{m}$ tema üldine Lie kolmiksüsteem $\left[{ }^{3,4}\right]$. Artiklis on sisse toodud reduktiivse struktuuri alamstruktuuri mōiste ning tõestatud, et niisuguste alamstruktuuride olemasolu on ekvivalentne alamsüsteemide leidumisega selle struktuuri üldises Lie kolmiksüsteemis. On uuritud loomulikult reduktiivsete r'umide täielikult geodeetiliste alammuutkondade ja Lie kolmiksüsteemide vahelisi seoseid. 


\section{A. FLJAISER}

\section{REDUCTIVE STRUCTURES AND LIE TRIPLE SYSTEMS}

Let $(\mathfrak{g}, \mathfrak{h}, \mathfrak{m})$ be a reductive structure $\left[{ }^{1,2}\right]$ on homogeneous space $G / H$. Following A. Sagle $\left[{ }^{4}\right]$, we make $\mathfrak{m}$ into general Lie triple system $\left[^{3}\right]$, assuming

$$
X \cdot Y=[X, Y]_{\mathfrak{m}} \text { and }[X, Y, Z]=(1 / 4)\left[[X, Y]_{\mathfrak{h}}, Z\right]
$$

for $X, Y, Z \in \mathfrak{m}$.

In the first part of this paper we introduce the notion of substructure of reductive structure as follows. Let $(\mathfrak{g}, \mathfrak{h}, \mathfrak{m})$ be a reductive structure on $G / H$. The subalgebra $\mathfrak{g}^{\prime} \subset \mathfrak{g}$ is called normal in $\mathfrak{g}\left[{ }^{6}\right]$ if

$$
\mathfrak{g}^{\prime}=\mathfrak{g}^{\prime} \cap \mathfrak{h}+\mathfrak{g}^{\prime} \cap \mathfrak{m} .
$$

The triple $\left(\mathfrak{g}^{\prime}, \mathfrak{h}^{\prime}, \mathfrak{m}^{\prime}\right)$ is called a reductive substructure of $(\mathfrak{g}, \mathfrak{h}, \mathfrak{m})$ if $\mathfrak{g}^{\prime}$ is a normal subalgebra in $\mathfrak{g}, \mathfrak{h}^{\prime}=\mathfrak{g}^{\prime} \cap \mathfrak{h}$ and $\mathfrak{m}^{\prime}=\mathfrak{g}^{\prime} \cap \mathfrak{m}$. The finding of such substructures, as it is shown, is equivalent to the finding of subsystems in the general Lie triple system $\mathrm{m}$. The special case (when $\mathfrak{g}^{\prime}$ is an ideal in $\mathfrak{g}$ ) is studied independently.

In the second part we consider naturally reductive isotropy - irreducible structures $\mathfrak{g}, \mathfrak{h}, \mathfrak{m})$ on $M=G / H$ and prove that every Lie triple system $\mathfrak{s} \subset \mathfrak{m}$ defines a totally geodesic symmetric submanifold $S=\pi \cdot \exp (\mathfrak{s})$ with respect to the natural connection corresponding to the given structure. Conversely if $S \subset M$ is a totally geodesic symmetric submanifold with respect to the natural connection of the given structure, then there is a Lie triple system $\mathfrak{s} \subset \mathfrak{m}$ with $S=\pi \cdot \exp (\mathfrak{s})$. This result generalizes a wellknown result of E. Cartan for symmetric spaces. 